\title{
ОБОБЩЕННЫЙ МЕТОД РЕШЕНИЯ ТИПОВОЙ ПРОФЕССИОНАЛЬНОЙ ЗАДАЧИ БАКАЛАВРОВ-СТРОИТЕЛЕЙ ПО ПРОЕКТИРОВАНИЮ ОБЬЕКТОВ ПРОФЕССИОНАЛЬНОЙ ДЕЯТЕЛЬНОСТИ
}

\author{
Соболева В.В. ${ }^{1}$
}

${ }^{1}$ ГАОУ АО ВО «Астраханский государственный архитектурно-строительный университет», Астрахань, еmail:veravsoboleva@yandex.ru

Подготовка высококвалифицированных инженеров, способных творчески подходить к решению сложных инженерных задач, является в настоящее время приоритетной задачей развития современного общества. Возникновение потребности в квалифицированных инженерах в области строительства приводит к необходиости поиска наиболее эффективных методов подготовки специалистов в технических вузах. Одним из наиболее успешных методов обучения является формирование в процессе обучения обобщенных методов решения типовых профессиональных задач. В статье предложен один из методов подготовки студентов, обучающихся по направлению «Строительство», профиль «Промышленное и гражданское строительство» к профессиональной деятельности на занятиях по физике. Предложенный метод позволяет сформировать обобщенные умения по проектированию объектов профессиональной деятельности с опорой на знания из общего курса физики. В статье уточнен «механизм» выделения типовых профессиональных задач бакалавров-строителей, выделены основные положения для формирования обобщенного метода, а также содержание обобщенных методов отдельных этапов проектирования объектов профессиональной деятельности, формируемых при обучении физике. На конкретном примере показан обобщенный метод одного из этапов проектирования, связанный с расчетом механических (прочностных) характеристик объекта профессиональной деятельности бакалавра-строителя.

Ключевые слова: профессиональная деятельность бакалавра-строителя, проектирование, типовая профессиональная задача, обобщенный метод решения, формирование метода.

\section{THE GENERALIZED METHOD OF SOLUTION OF THE STANDARD PROFESSIONAL TASK OF BACHELORS-BUILDERS OF PROJECTION OF OBJECTS OF PROFESSIONAL ACTIVITY}

\author{
Soboleva V.V.1

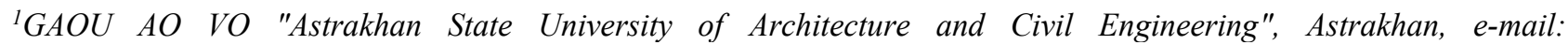 \\ veravsoboleva@yandex.ru
}

Training of the highly skilled engineers capable to approach creatively the solution of difficult engineering tasks is a priority problem of development of modern society now. Emergence of need for the qualified engineers in the field of construction results in need of search of the most effective methods of training of specialists for technical colleges. One of the most successful methods of training is formation in the course of training of the generalized methods of the solution of standard professional tasks. In article one of methods of training of the students studying in the direction "Construction", "Industrial and Civil Engineering" profile to professional activity at classes in physics is offered. The offered method allows to create the generalized abilities on design of objects of professional activity with a support on knowledge from the general course of physics. In article "mechanism" of allocation of standard professional tasks of bachelors-builders is specified, basic provisions for formation of the generalized method and also the maintenance of the generalized methods of separate design stages of the objects of professional activity formed when training in physics are allocated. On a concrete example the generalized method of one of design stages connected with calculation of mechanical (strength) characteristics of an object of professional activity of the bachelor-builder is shown.

Keywords: professional activity of the bachelor-builder, projection, standard professional problem, the generalized method of solution, formation of a method.

Анализ квалификационных характеристик выпускника по направлению подготовки 08.03 .01 «Строительство», сформулированных в Федеральном государственном
образовательном стандарте высшего образования (ФГОС ВО 3++) нового поколения 
свидетельствует о том, что одним из важнейших умений является проектирование объектов профессиональной деятельности. К объектам профессиональной деятельности будущего бакалавра-строителя относятся, например, промышленные и гражданские здания, гидротехнические и природоохранные сооружения, строительные конструкции и др. Проектирование объектов профессиональной деятельности является практически значимой, типовой профессиональной задачей для выпускника строительного вуза. Метод решения типовой профессиональной задачи основывается на знаниях естественно-научных, общепрофессиональных и специальных дисциплин.

Курс физики в техническом вузе составляет основополагающую базу, на которой строится общепрофессиональная и специальная подготовка бакалавров-строителей. Анализ учебных планов направления «Строительство» различных классических и технических вузов показал, что физика изучается в течение двух семестров и в среднем составляет 252 часа. Физические знания применяются для расчетов нагрузок на строительный объект; значений прочностных, деформационных и других физико-механических характеристик строительных материалов и грунтов; выполнения теплотехнических расчетов; расчета и оценки основных световых характеристик помещения и др. Все эти виды деятельности являются крайне необходимыми для проектирования объектов профессиональной деятельности.

Чтобы профессиональные задачи бакалавра-строителя включить в цели обучения физике, необходимо дополнить эти задачи обобщенными методами решения. Обобщенные методы решения задач используются при решении большого круга практически значимых задач [1-3]. В частности, сформированный обобщенный метод проектирования отдельного элемента строительной конструкции на занятиях по физике может быть использован в дальнейшем при изучении общетехнических и специальных дисциплин для проектирования любого инженерного сооружения в целом.

\section{Цель исследования}

Целью нашего исследования является разработка методики обучения проектированию объектов профессиональной деятельности студентов направления «Строительство», используя обобщенные методы решения типовых профессиональных задач, с применением знаний из курса физики.

\section{Материал и методы исследования}

Для выделения типовых профессиональных задач бакалавров-строителей воспользуемся «механизмом», предложенным Г.П. Стефановой [4]. Содержание данного «механизма» включает:

1) анализ практически значимых ситуаций, видов деятельности бакалавра-строителя, дипломных и курсовых проектов студентов, обучающихся по профилю «Промышленное и 
гражданское строительство», с целью выявления типовых профессиональных задач, решаемых с опорой на физические знания;

2) конкретизацию объектов профессиональной деятельности с учетом знаний курса физики. При этом следует иметь в виду, что конструкция сложной формы требует выделения отдельных элементов и сведения их к простейшим типам: брус (стержень, балка, стержневые системы), пластина (плита), оболочка, массивное тело;

3) выявление типовых профессиональных задач будущего бакалавра-строителя, в которых используются знания из курса физики.

Данный «механизм» позволил нам выделить основную типовую профессиональную задачу, связанную с проектированием объектов профессиональной деятельности. Оказалось, что эта задача включает следующие этапы, выполняемые на основе физических знаний: расчет механических характеристик объектов профессиональной деятельности или его отдельных элементов; расчет теплофизических характеристик строительных конструкций или его отдельных элементов; расчет световых характеристик зданий и сооружений.

В процессе выделения обобщенных методов решения типовых профессиональных задач бакалавра-строителя, содержащих отдельные этапы по проектированию конструкций, будем руководствоваться следующими положениями.

1. Деятельность по проектированию объектов профессиональной деятельности представляет собой целенаправленную систему взаимосвязанных элементов, объединенных единым мотивом и в совокупности обеспечивающих достижение конечной цели деятельности (разработка проектной документации строительного объекта), в состав которой они входят [5]. Взаимосвязь этих элементов обеспечивается за счет того, что результаты, полученные при изучении физики, передаются на следующие изучаемые дисциплины с постепенным усложнением и укрупнением этапов деятельности по проектированию. В результате последовательное выполнение отдельных этапов, через решение типовых профессиональных задач, приводит студентов к конечному результату деятельности проектированию объекта профессиональной деятельности.

2. Любая деятельность по достижению поставленной цели состоит из трех этапов (ориентировочный, исполнительный и контрольно-корректировочный). На ориентировочном этапе студент определяет цель, выделяет объект и его свойства, а также составляет программу действий по преобразованию выделенного объекта в конечный объект с заданными свойствами. На втором этапе (исполнительном), используя разработанную программу действий, студент создает конечный объект с заданными свойствами и фиксирует информацию о его свойствах. На следующем (контрольно-корректировочном) этапе студенты сравнивают свойства конечного объекта с заданными, указанными в цели, 
выявляют несоответствия и при необходимости вносят коррективы в разработанную программу действий.

Нами выделено содержание трех этапов типовой профессиональной задачи «Проектирование объектов профессиональной деятельности». Выделим обобщенное содержание одного из этапов решения типовых профессиональных задач бакалаврастроителя, обучение которым осуществляется на занятиях по физике.

Содержание 1 этапа. Расчет механических характеристик объектов профессиональной деятельности или его отдельных элементов.

Выделяем цель деятельности: рассчитать механические характеристики объектов профессиональной деятельности или его отдельного элемента.

1. На исполнительном этапе устанавливаем, что в формулировке цель деятельности указана - рассчитать; конечным продуктом является значение механической характеристики объекта; свойства конечного продукта указаны - механические характеристики, описывающие объект профессиональной деятельности или его отдельный элемент.

2. Выделяем действия, которые необходимо выполнить на ориентировочном этапе: выделить объект профессиональной деятельности или его отдельный элемент; установить вид нагрузки (распределенная, сосредоточенная, постоянная, временная, статическая и т.д.), действующий на выделенный объект профессиональной деятельности или его отдельный элемент; указать физические явления и установить виды деформации; изобразить графическую модель поведения проектируемого объекта профессиональной деятельности или его отдельного элемента; установить физические величины, характеризующие данный вид деформации; записать физические законы, описывающие поведение объектов профессиональной деятельности или его отдельного элемента в заданных условиях; решить полученную систему уравнений и найти расчетное значение искомой физической величины.

3. Выделяем действие контрольно-корректировочного этапа: сравнить расчетное значение с нормативным согласно техническим условия и нормам проектирования.

Данному обобщенному методу решения типовой профессиональной задачи № 1 целесообразно обучать студентов при изучении раздела физики «Физические основы механики», включая «Механику упругих тел».

\section{Результаты исследования и их обсуждение}

Конкретизируем метод выполнения первого этапа проектирования, связанного с расчетом механических характеристик объекта профессиональной деятельности, опираясь на обобщенный метод.

Задача. В здании склада железобетонная балка опирается на пилястру и кирпичную колонну (рис. 1). Определить максимальный изгибающий момент железобетонной балки от 
собственного веса, если полная нагрузка, которую может выдержать балка, без учета собственного веса равна 600 кН/м. Сечение балки 200x400 мм. Удельный вес железобетона 25 кН/м³. Необходимые размеры на чертеже даны в миллиметрах.

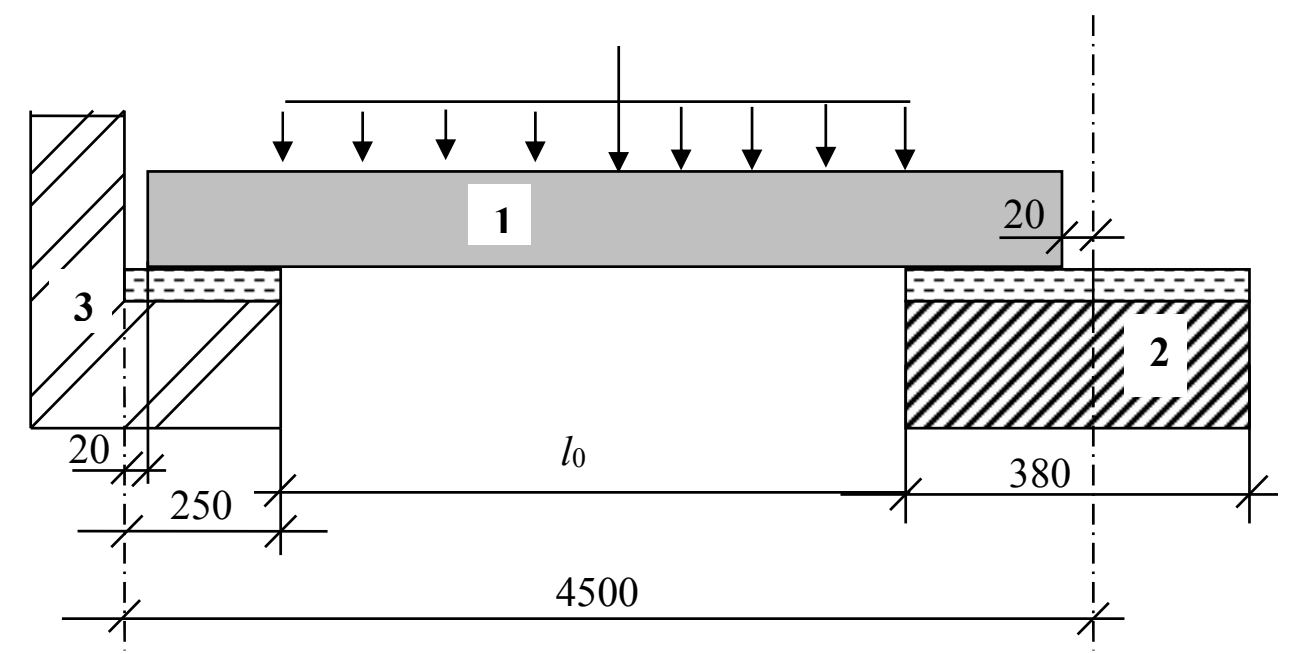

Рис. 1. Схема опирания балки:

1 - железобетонная балка; 2 - кирпичная колонна; 3 - пилястра

\section{Метод решения}

В первом действии обобщенного метода необходимо выделить объект профессиональной деятельности или его отдельного элемента. В данной задаче таким объектом является железобетонная балка, которая опирается на пилястру и кирпичную колонну.

Вторым действием обобщенного метода является установление вида нагрузки (распределенная, сосредоточенная, постоянная, временная, статическая и т.д.), действующей на выделенный объект профессиональной деятельности или его отдельный элемент. Согласно условию задачи, железобетонная балка испытывает нагрузку от собственного веса. Данную нагрузку необходимо учитывать в течение всего периода эксплуатации, поэтому она является постоянной. В практике инженерных расчетов нагрузка от собственного веса конструкций часто приводится к поверхностным нагрузкам, а они в свою очередь являются распределенными по некоторой поверхности. При расчете стержней распределенная нагрузка приводится к линейной нагрузке, распределенной по всей длине стержня. Поэтому в условии данной задачи нагрузка линейная распределенная и постоянная. Ее интенсивность определяется силой, отнесенной к единице длины объекта. 
Третье действие обобщенного метода заключается в указании физических явлений и установлении вида деформации. Под действием постоянной нагрузки железобетонная балка деформируется, т.е. подвергается деформации на изгиб. В четвертом действии обобщенного метода необходимо изобразить графическую модель поведения проектируемого объекта профессиональной деятельности или его отдельного элемента.

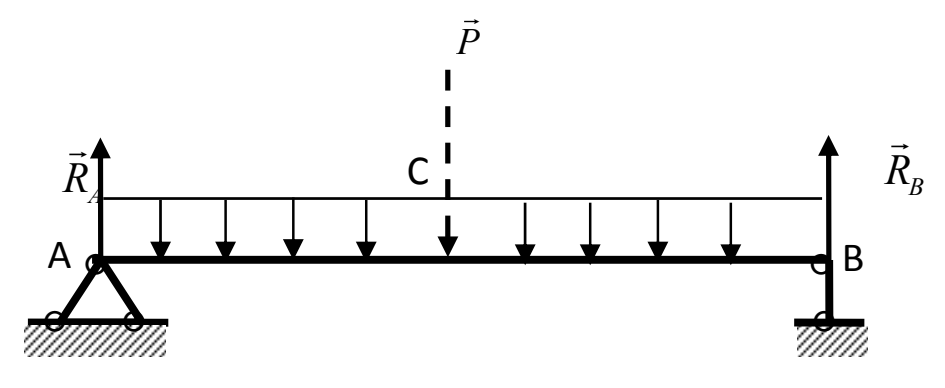

Рис. 2. Расчетная схема опирания балки

Расчет конструкции с учетом всех механических свойств твердого тела, точных геометрических размеров и строгого взаимодействия отдельных элементов является очень сложным. Поэтому перед решением задачи сделаем расчетную схему - упрощенную идеализированную схему, которая отображает наиболее существенные особенности реального объекта (конструкции или отдельного элемента), определяющие его поведение под нагрузкой (рис. 2). В пятом действии обобщенного метода необходимо установить физические величины, характеризующие данный вид деформации: а) момент силы; б) вес тела; в) сила давления; г) удельный вес балки. В шестом действии обобщенного метода необходимо записать физические законы, описывающие поведение объектов профессиональной деятельности или его отдельного элемента конструкции в заданных условиях.

Условие равновесия системы: $\sum_{i=1}^{n} \vec{M}_{i}=0$.

Конкретизируем условие равновесия относительно точки А и точки В:

$R_{B} \cdot l=P \cdot \frac{l}{2}, \quad R_{A} \cdot l=P \cdot \frac{l}{2}$.

Следовательно, $R=R_{A}=R_{B}=\frac{P}{2}$.

Мысленно проведем через балку плоскость, которая делит балку на две равные части. В каждой части будут действовать силы, характеризующие взаимодействия частей АС и СВ. Отбросим правую часть балки. Жестко закрепляем в месте отсечения и определим максимальный изгибающий момент в этой точки от действия перегрузки и реакции опоры R. 
Предварительно равномерно распределенную нагрузку заменяем на сосредоточенную.

На рисунке 3 нагрузка $\mathrm{Q}=\mathrm{P} / 2$ является условной заменой равномерно распределенной нагрузки на участке $/ / 2$. Располагаем оси координат в центре тяжести сечения (рис. 3). Записываем уравнения равновесия железобетонной балки относительно точки А (рис. 3).

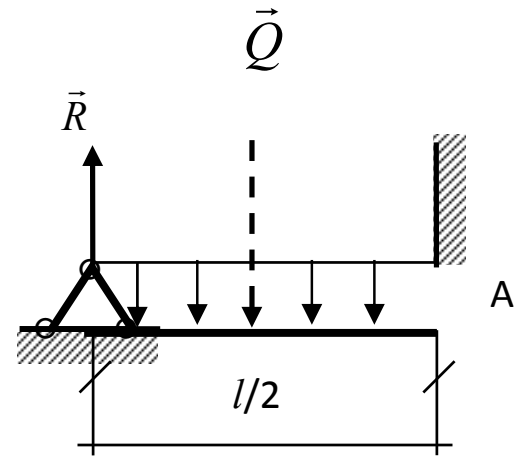

Рис. 3. Участок конструкции под нагрузкой

$$
M_{\max }=\frac{P}{2} \cdot \frac{l}{2}-\frac{P}{2} \cdot \frac{l}{4}=\frac{P}{2}\left(\frac{l}{2}-\frac{l}{4}\right)=\frac{P \cdot l}{8} .
$$

Если учесть, что произведение силы на длину балки это есть нагрузка, то

$$
M_{\max }=\frac{(q \cdot l) \cdot l}{8}=\frac{q l^{2}}{8} .
$$

В седьмом действии обобщенного метода необходимо решить полученную систему уравнений и найти расчетное значение искомой физической величины.

Для простоты расчета не будем учитывать экономические, социальные и экологические последствия, которые могут возникать в результате аварий, т.е. коэффициентом надежности по ответственности пренебрегаем. В этом случае сила, действующая на 1 метр балки с учетом собственного веса:

$$
q_{l}=\mathrm{F}_{l \text { pез }}=2000 \mathrm{H} / \mathrm{M} \text {. }
$$

Определим давление на 1 метр от собственного веса балки:

$$
F_{l_{\text {дав }}}=\frac{F_{\text {дав }}}{l}=b \cdot h \cdot \gamma=2000 \mathrm{H} / \mathrm{M},
$$

где $F_{\text {дав }}=\rho \cdot V \cdot g=\rho \cdot b \cdot h \cdot l \cdot g$ - сила давления собственного веса, $\gamma=\rho \cdot g$ - удельный вес балки. Из рисунка определяем расчетную длину балки:

$$
l_{0}=l-(20+20)-\frac{l_{\mathrm{K}}-l_{1}}{2}-\frac{l_{n}-l_{1}}{2}-=4500-40-\frac{250-20}{2}-\frac{380-20}{2} \approx 4200 \mathrm{MM}=4,2 \mathrm{M}
$$

Проведем расчет результирующего значения поперечных сил относительно оси, проходящей через центр балки: 


$$
\begin{gathered}
F_{\text {рез }}=q_{l} \cdot \frac{l_{0}}{2}=2000 \cdot \frac{4,2}{2}=4200 \mathrm{H}=4,2 \kappa \mathrm{H} \\
q=P-F_{l \text { рез }}=600 \cdot 10^{3}-2 \cdot 10^{3}=598 \cdot 10^{3} \mathrm{H} / \mathrm{M}
\end{gathered}
$$

Следовательно, максимальный изгибающий момент

$$
M_{\max }=\frac{598 \cdot 10^{3} \cdot 4,2^{2}}{8}=1318,9 \cdot 10^{3} \mathrm{H} \cdot \mathrm{M}
$$

На контрольно-корректировочном этапе деятельности выполняется сравнение расчетного значения с нормативным согласно техническим условия и нормам проектирования.

\section{Выводы}

Основная типовая профессиональная задача бакалавров-строителей «Проектирование объектов профессиональной деятельности» содержит отдельные этапы, обучение которым возможно и целесообразно осуществлять на занятиях по физике (расчет механических (прочностных) характеристик объектов профессиональной деятельности или его отдельных элементов; расчет теплофизических характеристик строительных конструкций или его отдельных элементов; расчет световых характеристик зданий и сооружений). Обобщенное содержание по выполнению каждого этапа, с опорой на физические знания, а также разработанные специальные дидактические средства, необходимые для усвоения этих этапов, позволяют повысить качество подготовки будущих бакалавров-строителей к проектированию строительного объекта любой сложности.

\section{Список литературы}

1. Стефанова Г.П. Подготовка учащихся к практической деятельности при обучении физике: пособие для учителя. Астрахань: Изд-во Астрахан. гос. пед. ун-та, 2001. 184 с.

2. Соболева В.В. Теоретические основы метода сквозного проектирования объектов профессиональной деятельности инженера-строителя при изучении курса общей физики // Современные проблемы науки и образования. 2012. №3. [Электронный ресурс]. URL: http://science-education.ru/ru/article/view?id=6227 (дата обращения: 20.10.2018).

3. Крутова И.А., Валишева А.Г. Обучение студентов обобщенным методам решения профессиональных задач инженера // Вестник ТГПУ. 2011. Выпуск 2 (104). С.95-99.

4. Скрипко Л.П. Формирование методов решения профессиональных задачслушателей института морских технологий, энергетики и транспорта // Вестник АГТУ. 2016. №1. С. 71 75.

5. Смирнов В.В. Методическая система формирования обобщенных методов проведения 
физических экспериментальных исследований у студентов физико-математического направления подготовки: дис. ... док. пед. наук. Волгоград, 2012. 45 с. 